\title{
Spectral Analysis for Intrinsic Time Processes
}

\author{
TAKAHIDE ISHIOKA, SHUNSUKE KAWAMURA, \\ TOMOYUKI AMANO AND MASANOBU TANIGUCHI \\ Department of Pure and Applied Mathematics, Graduate School of Fundamental \\ Science and Engineering, Waseda University, Japan
}

\begin{abstract}
This paper discusses estimation procedure for the spectral density of intrinsic time processes because there has been no argument of the spectral analysis for subordinated processes. Such processes have been proposed in a variety of contexts to describe asset price behavior. They are used when the movement of prices is tied to the number of market transactions, trading volume or the more illusive concept of information arrival. We develop the asymptotic theory for an estimated spectral density of intrinsic time processes and elucidate the asymptotics, which show some interesting structures. Also, numerical studies are given to confirm the results.
\end{abstract}

Keywords: Spectral density; Intrinsic time; Subordinated process; Local Whittle likelihood; Irregularly spaced data; Statistical asymptotic theory

Mathematics Subject Classification: 62M15.

\section{Introduction}

While most of time series are recorded at regularly spaced intervals, there are many cases where observations are irregularly spaced. For example, until a few years ago most empirical studies involved daily, weekly, or monthly time series. As high frequency data becomes more easily available it is possible to study how financial markets evolve in real time. But unlike daily, weekly or monthly series, quote or tick-based data are by their very nature irregularly spaced. If time series data on factory output covers a period with a strike, the serial correlation in the series might best be captured using a time scale based on the days during which the plant was operating, rather than simply units of calender time. In such cases, we will focus on situations where a subordinated process framework is adopted. 
The idea originated in the work by Mandelbrot and Taylor (1967), Clark (1973) among others, one may think of asset price movements as the realization of a process $Y_{t}=Y_{Z_{t}}^{*}$ where $\left\{Z_{t}\right\}$ is a directing process and $\left\{Y_{z}^{*}\right\}$ is an intrinsic time process. This positive nondecreasing stochastic process $\left\{Z_{t}\right\}$ can, for instance, be thought as related to the arrival of information. In practice, one uses nondecreasing processes like either the number of transactions or the cumulated trading volume, or the cumulated volatility as directing processes. Furthermore, Ghysels, Gouriéroux and Jasiak (1998) describe the comprehensive treatment of the stochastic process theory and statistical estimation of subordinated processes. But there has been no argument of the spectral analysis for subordinated processes.

In this paper, we develop estimation and asymptotic theory of the spectral density function $f^{*}(\lambda)$ for intrinsic time processes. Because $\left\{Y_{z}^{*}\right\}$ is not observable, we have to estimate $f^{*}(\lambda)$ from observations of calender time process $\left\{Y_{t}\right\}$. For this we use a relation between spectral densities for calender time process and intrinsic time process, and introduce a local Whittle likelihood. Based on it we propose an estimator of the spectral density of $\left\{Y_{z}^{*}\right\}$, and provide the asymptotic theory. Numerical studies are given, and show some interesting features of the asymptotics of our estimator.

This paper is organized as follows. Section2 presents a brief review of subordinated processes and their properies. Further, we mention a relation of spectral structure between calender time process and intrinsic time process. In Section3, we introduce a local Whittle likelihood from the observations of $\left\{Y_{t}\right\}$, and propose an estimator of the spectral density of $\left\{Y_{z}^{*}\right\}$. The asymptotics are elucidated in terms of the spectral structure and a characteristic function of the directing process. Numerical studies are given, and they illuminate an intersting feature of our estimator. The proofs of theorems are given in Section5.

Throughout this paper we use the following notations. $\mathcal{N}$ is the set of all positive integers, $\mathcal{Z}$ is the set of all integers and $\mathcal{R}$ is the set of all real numbers. Moreover $[x]$ is the integer part of a real number $x$ and $\|\boldsymbol{a}\|$ is the Euclidean norm of a vector $\boldsymbol{a}$.

\section{Subordinated Processes}

In this section we describe the properties of the process of interest, when it evolves in calender time and in intrinsic(or operational) time. We first introduce three fundamental processes and some notations:

(1) directing process

The time changing process, called the directing process by Clark (1973), associates the operational scale with the calender time. It is a real-valued process defined by

$$
Z: t \in \mathcal{R} \longrightarrow Z_{t} \in \mathcal{R}
$$

(2) intrinsic time process

The process of interest evolving in the operational time is denoted by 


$$
Y^{*}: z \in \mathcal{R} \longrightarrow Y_{z}^{*} \in \mathcal{R} .
$$

(3) calender time process

Finally we may deduce the process in calender time $t \in \mathcal{R}$ by considering

$$
Y_{t}=Y^{*} \circ Z_{t}=Y_{Z_{t}}^{*} .
$$

The introduction of a time scaling process is interesting when the probabilistic properties of the process of interest become simpler.

As example of the above setting, the price series $Y_{t}$ for cotton futures evolves at different rates during identical intervals of time. The number of individual effects added to together to give the price chance during a day is variable. In this case the directing process $Z_{t}$ would be the cumulative trading volume up to time $t$. It explains the assumptions below which ensure that all the links between the two processes $\left(Y_{t}\right),\left(Z_{t}\right)$ in calender time come from the time deformation.

Assumption 1. The two processes $Z=\left\{Z_{t}: t \in \mathcal{R}\right\}$ and $Y^{*}=\left\{Y_{z}^{*}: z \in \mathcal{R}\right\}$ are mutually indepedent.

As usual for time series analysis we study the second order properties of the processes $Y$ and $Y^{*}$. Assuming that both processes are second order integrable, we denote the first order moment by

$$
\left\{\begin{array}{l}
m(t) \equiv E\left(Y_{t}\right), t \in \mathcal{R} \\
m^{*}(z) \equiv E\left(Y_{z}^{*}\right), z \in \mathcal{R}
\end{array}\right.
$$

and the autocovariance functions

$$
\left\{\begin{array}{l}
\gamma(t, h) \equiv E\left[\left(Y_{t}-E Y_{t}\right)\left(Y_{t+h}-E Y_{t+h}\right)\right], t \in \mathcal{R}, h \in \mathcal{R} \\
\gamma^{*}\left(z_{0}, z\right) \equiv E\left[\left[Y_{z_{0}}^{*}-E\left(Y_{z_{0}}^{*}\right)\right]\left[Y_{z+z_{0}}^{*}-E\left(Y_{z+z_{0}}^{*}\right)\right]\right], z_{0} \in \mathcal{R}, z \in \mathcal{R}
\end{array}\right.
$$

From the definition of the time deformed process,

$$
\begin{aligned}
m(t) & =E\left(Y_{t}\right)=E\left[E\left(Y_{Z_{t}}^{*} \mid Z_{t}\right)\right] \\
\gamma(t, h) & =E\left(Y_{t} Y_{t+h}\right)-\left(E Y_{t}\right)\left(E Y_{t+h}\right)=E\left[E\left(Y_{t} Y_{t+h} \mid Z_{t}, Z_{t+h}\right)\right]-\left(E Y_{t}\right)\left(E Y_{t+h}\right) .
\end{aligned}
$$

We next make some fundamental assumptions on $Y^{*}$ and $Z$.

Assumption 2. $Y^{*}$ is second order stationary, i.e., $m^{*}(z)=m^{*}$, for all $z \in \mathcal{R}$, and $\gamma^{*}\left(z_{0}, z\right)=\gamma^{*}(z)$, for all $z_{0}, z \in \mathcal{R}$.

Assumption 3. The directing process has strongly stationary increments, i.e., for $h, t \in \mathcal{R}$, the distribution of $\Delta_{h} Z_{t}=Z_{t+h}-Z_{t}$ is independent of $t$.

From Assumptions 2 and 3, we have second order stationarity of the processes $Y$, i.e.,

$$
m(t)=m^{*}, \quad \gamma(t, h)=E\left[\gamma^{*}\left(\Delta_{h} Z_{t}\right)\right] \equiv \gamma(h), \text { (say). }
$$


We suppose that $Y$ and $Y^{*}$ have spectral densities $f(\lambda)$ and $f^{*}(\lambda)$, respectively. Then we have the following relationship:

$$
\gamma(z)=\int_{-\infty}^{\infty} e^{i z \lambda} f(\lambda) d \lambda \quad, \quad \gamma^{*}(z)=\int_{-\infty}^{\infty} e^{i z \lambda} f^{*}(\lambda) d \lambda .
$$

If Assumptions 1-3 hold, it is seen that

$$
\begin{aligned}
\gamma(h) & =E\left\{\gamma^{*}\left(Z_{t}, Z_{t+h}-Z_{t}\right)\right\}=E\left\{\gamma^{*}\left(Z_{t+h}-Z_{t}\right)\right\} \\
& =\int_{-\infty}^{\infty} E\left\{e^{i \Delta_{h} Z_{t} \lambda}\right\} f^{*}(\lambda) d \lambda,
\end{aligned}
$$

hence,

$$
\int_{-\infty}^{\infty} e^{i h \lambda} f(\lambda) d \lambda=\int_{-\infty}^{\infty} E\left\{e^{i \Delta_{h} Z_{t} \lambda}\right\} f^{*}(\lambda) d \lambda
$$

In what follows we impose the following.

Assumption 4.

$$
E\left\{e^{i\left(\Delta_{h} Z_{t}\right) \lambda}\right\}=\exp (i h \lambda) \varphi(\lambda)
$$

where $\varphi(\lambda)$ is a function which is independent of $h$.

Then, we can have the relation between $f(\lambda)$ and $f^{*}(\lambda)$ :

$$
f(\lambda)=\varphi(\lambda) f^{*}(\lambda) .
$$

We provide a few examples of the distributions of $\Delta_{h} Z_{t}$ satisfying (2.7)

Example 1. If $\Delta_{h} Z_{t} \sim N\left(h, \sigma^{2}\right)$, then

$$
E\left\{e^{i\left(\Delta_{h} Z_{t}\right) \lambda}\right\}=\exp (i h \lambda) \exp \left(-\frac{\sigma^{2}}{2} \lambda^{2}\right) .
$$

Therefore,

$$
\varphi(\lambda)=\exp \left(-\frac{\sigma^{2}}{2} \lambda^{2}\right)
$$

Example 2. If $\left(\Delta_{h} Z_{t}\right) \sim \operatorname{Exp}(h, \theta)$, then

Therefore,

$$
E\left\{e^{i\left(\Delta_{h} Z_{t}\right) \lambda}\right\}=\exp (i h \lambda)\left(1-\frac{i \lambda}{\theta}\right)^{-1} .
$$

$$
\varphi(\lambda)=\left(1-\frac{i \lambda}{\theta}\right)^{-1}
$$

Example 3. If $\left(\Delta_{h} Z_{t}\right)$ is distributed as a stable distribution $S_{\alpha}(\sigma, \beta, h)$ characterized by the characteristic function

$$
E\left\{e^{i\left(\Delta_{h} Z_{t}\right) \lambda}\right\}= \begin{cases}\exp (i h \lambda) \exp \left\{-\sigma^{\alpha}|\lambda|^{\alpha}\left(1-i \beta \operatorname{sign}(\lambda) \tan \frac{\pi \alpha}{2}\right)\right\} & \text { if } \quad \alpha \neq 1 \\ \exp (i h \lambda) \exp \left\{-\sigma|\lambda|\left(1+i \beta \frac{2}{\pi} \operatorname{sign}(\lambda) \log \lambda\right)\right\} & \text { if } \quad \alpha=1\end{cases}
$$


Then,

$$
\varphi(\lambda)= \begin{cases}\exp \left\{-\sigma^{\alpha}|\lambda|^{\alpha}\left(1-i \beta \operatorname{sign}(\lambda) \tan \frac{\pi \alpha}{2}\right)\right\} & \text { if } \quad \alpha \neq 1 \\ \exp \left\{-\sigma|\lambda|\left(1+i \beta \frac{2}{\pi} \operatorname{sign}(\lambda) \log \lambda\right)\right\} & \text { if } \quad \alpha=1 .\end{cases}
$$

The probability density function of $S_{\alpha}(\sigma, \beta, h)$ does not admit explicit expression except for the following special cases:

(i) $\alpha=2, \beta=0$, the normal distribution,

(ii) $\alpha=1, \beta=0$, the Cauchy distribution,

(iii) $\alpha=0.5, \beta=1$, the Levy distribution.

It can be skewed to the left or right, depending on the sign of $\beta$, and is symmetric when $\beta=0$. If $\alpha<2$, it has fatter tails than the normal distribution and the $p$ th absolute moment does not exist for any $p>\alpha$, and in particular the variance is infinite. If $1<\alpha<2$, the probability density function has no closed form, and it has to be calculated numerically by Fourier inverse transformation of (2.11). When $\alpha>1$, the mean exists and equals $h$. Unless $\beta=0$, the mean is different from the median.

\section{The Estimation Procedure}

In this section we estimate $f^{*}(\lambda)$ from a partial realization $Y_{1}, \ldots, Y_{n}$, under assumption that $\varphi(\lambda)$ is known. For all $l \in \mathcal{Z}$, we can rewrite $\gamma(l)$ as

$$
\gamma(l)=\int_{-\pi}^{\pi} \exp (i l \lambda) \tilde{f}(\lambda) d \lambda,
$$

where $\tilde{f}(\lambda)$ is the spectral density of the discrete time process $Y_{n}, n \in \mathcal{Z}$, which is representable as $\tilde{f}(\lambda)=\sum_{j=-\infty}^{\infty} f(\lambda+2 \pi j$ ) (see Hannan (1970), p.45). Correspondingly it follows from $(2.8)$ that

$$
\tilde{f}(\lambda)=\sum_{j=-\infty}^{\infty} \varphi(\lambda+2 \pi j) f^{*}(\lambda+2 \pi j)
$$

Similarly, if we set $\tilde{f}^{*}(\lambda)=\sum_{j=-\infty}^{\infty} f^{*}(\lambda+2 \pi j)$, then it holds that

$$
\gamma^{*}(l)=\int_{-\pi}^{\pi} \exp (i l \lambda) \tilde{f}^{*}(\lambda) d \lambda .
$$

We can understand that $\tilde{f}^{*}(\lambda)$ is the spectral density of the discrete time process $Y_{n}^{*}, n \in \mathcal{Z}$. For a sufficiently large fixed $M \in \mathcal{N}$, we partition the interval $[0, \pi]$ into $M$ subintervals. For an arbitrarily chosen $\lambda_{0} \in[0, \pi]$, let $I_{M}^{0}=$ $\left[\lambda_{0}-\frac{2 \pi}{n} L, \lambda_{0}+\frac{2 \pi}{n} L\right]$ where $L=\left[\frac{n}{4 M}\right]$. We may suppose that if $f^{*}$ is smooth,

$$
f^{*}(\lambda+2 \pi j) \approx f^{*}\left(\lambda_{0}+2 \pi j\right) \equiv \theta_{j}(\text { say }), \text { for } \lambda \in I_{M}^{0} .
$$

In view of Example 1-3, in many cases, we may assume

Assumption 5.

$$
\varphi\left(\lambda_{0}+2 \pi j\right) \rightarrow 0(j \rightarrow \infty)
$$


Let $\tilde{f}_{M, \theta_{M}}\left(\lambda_{0}\right)=\sum_{j=-M}^{M} \varphi\left(\lambda_{0}+2 \pi j\right) \theta_{j}=\boldsymbol{\varphi}_{M}\left(\lambda_{0}\right)^{\prime} \boldsymbol{\theta}_{M}$, where $\boldsymbol{\varphi}_{M}\left(\lambda_{0}\right)=$ $\left(\varphi\left(\lambda_{0}-2 \pi M\right), \cdots, \varphi\left(\lambda_{0}\right), \cdots, \varphi\left(\lambda_{0}+2 \pi M\right)\right)^{\prime}, \boldsymbol{\theta}_{M}=\left(\theta_{-M}, \cdots, \theta_{0}, \cdots, \theta_{M}\right)^{\prime}$. For the observed stretch $Y_{1}, \cdots, Y_{n}$, the periodgram is given by

$$
I_{n}^{Y}(\lambda)=\frac{1}{2 \pi n}\left|\sum_{t=1}^{n} Y_{t} e^{i t \lambda}\right|^{2} .
$$

A local Whittle likelihood around $\lambda_{0}$ is introduced by

$$
D\left(\lambda_{0}, \tilde{f}_{M, \theta_{M}}, I_{n}^{Y}\right)=\frac{2 \pi}{L} \sum_{k=-L}^{L}\left[\log \tilde{f}_{M, \theta_{M}}\left(\lambda_{0}+\frac{2 \pi k}{n}\right)+\frac{I_{n}^{Y}\left(\lambda_{0}+\frac{2 \pi k}{n}\right)}{\tilde{f}_{M, \theta_{M}}\left(\lambda_{0}+\frac{2 \pi k}{n}\right)}\right] .
$$

Then the Whittle estimator of $\boldsymbol{\theta}_{M}$ is given by

$$
\hat{\boldsymbol{\theta}}_{M}=\left(\hat{\theta}_{-M}, \cdots, \hat{\theta}_{0}, \cdots, \hat{\theta}_{M}\right)^{\prime} \equiv \arg \min _{\theta_{M}} D\left(\lambda_{0}, \tilde{f}_{M, \theta_{M}}, I_{n}^{Y}\right) .
$$

Hence we can propose estimators of $\tilde{f}\left(\lambda_{0}\right)$ and $\tilde{f}^{*}\left(\lambda_{0}\right)$ by

$$
\begin{aligned}
& \hat{\tilde{f}}_{M, \theta_{M}}\left(\lambda_{0}\right)=\boldsymbol{\varphi}_{M}\left(\lambda_{0}\right)^{\prime} \hat{\boldsymbol{\theta}}_{M}, \\
& \hat{\tilde{f}}_{M, \theta_{M}}^{*}\left(\lambda_{0}\right)=\sum_{j=-M}^{M} \hat{\theta}_{j}
\end{aligned}
$$

respectively. To develop the asymptotic estimation theory for $\hat{\boldsymbol{\theta}}_{M}$ and $\hat{\tilde{f}}_{M, \theta_{M}}^{*}\left(\lambda_{0}\right)$, we introduce the following assumption.

Assumption 6 .

(a) for any continuous functions $g_{j}(\lambda)$

$$
\int_{-\pi}^{\pi} g_{j}(\lambda) I_{n}^{Y}(\lambda) d \lambda \stackrel{p}{\rightarrow} \int_{-\pi}^{\pi} g_{j}(\lambda) \tilde{f}(\lambda) d \lambda, \quad j=1, \cdots, q
$$

(b) the quantities

$$
\sqrt{n} \int_{-\pi}^{\pi}\left\{I_{n}^{Y}(\lambda) d \lambda-\tilde{f}(\lambda)\right\} g_{j}(\lambda) d \lambda
$$

have, asymptotically, a normal distribution with zero mean vector and covariance matrix $V$ whose $(j, l)$ element is

$$
\begin{aligned}
V_{j l}=\left\{4 \pi \int_{-\pi}^{\pi}\right. & {\left[f(\lambda)^{2} g_{j}(\lambda) g_{l}(\lambda)\right] d \lambda } \\
& \left.+2 \pi \iint_{-\pi}^{\pi}\left[g_{j}\left(\lambda_{1}\right) \cdot g_{l}\left(\lambda_{2}\right)\right] \tilde{Q}^{Y}\left(-\lambda_{1}, \lambda_{2},-\lambda_{2}\right) d \lambda_{1} d \lambda_{2}\right\}
\end{aligned}
$$


where $\widetilde{Q}^{Y}\left(w_{1}, w_{2}, w_{3}\right)=\frac{1}{(2 \pi)^{3}} \sum_{t_{1}, t_{2}, t_{3}=-\infty}^{\infty} \exp \left\{-i\left(w_{1} t_{1}+w_{2} t_{2}+w_{3} t_{3}\right)\right\} Q^{Y}\left(t_{1}, t_{2}, t_{3}\right)$, with $Q^{Y}\left(t_{1}, t_{2}, t_{3}\right)=\operatorname{cum}\left\{Y_{0}, Y_{t_{1}}, Y_{t_{2}}, Y_{t_{3}}\right\}$.

Remark 1. This assumption is very natural and not restrictive. Hosoya and Taniguchi (1982) established (a) and (b) for the case when $\left\{Y_{t}\right\}$ is a very general class of non-Gaussian linear processes.

We now derive the asymptotic distribution of $\hat{\boldsymbol{\theta}}_{M}$. Let

$$
\underline{\boldsymbol{\theta}}_{M}=\arg \min _{\theta_{M}} D\left(\lambda_{0}, \tilde{f}_{M, \theta_{M}}, \tilde{f}\right) .
$$

Then we have,

Theorem 1. If $M$ is a sufficiently large fixed integer and if

$$
M_{f}=4 M \int_{\lambda_{0}-\frac{\pi}{2 M}}^{\lambda_{0}+\frac{\pi}{2 M}}\left[\frac{\partial^{2}}{\partial \boldsymbol{\theta}_{M} \partial \boldsymbol{\theta}_{M}^{\prime}} \tilde{f}_{M, \theta_{M}}(\lambda)^{-1} \tilde{f}(\lambda)+\frac{\partial^{2}}{\partial \boldsymbol{\theta}_{M} \partial \boldsymbol{\theta}_{M}^{\prime}} \log \tilde{f}_{M, \theta_{M}}(\lambda)\right] d \lambda
$$

is a nonsingular matrix, then

$$
\sqrt{n}\left(\hat{\boldsymbol{\theta}}_{M}-\underline{\boldsymbol{\theta}}_{M}\right) \stackrel{d}{\rightarrow} N\left(\mathbf{0}, M_{f}^{-1} \widetilde{V} M_{f}^{-1}\right)
$$

where $\widetilde{V}=\left\{\widetilde{V}_{j l}\right\}$ is a matrix such that

$$
\begin{gathered}
\widetilde{V}_{j l}=16 M^{2}\left[4 \pi \int_{\lambda_{0}-\frac{\pi}{2 M}}^{\lambda_{0}+\frac{\pi}{2 M}}\left\{\tilde{f}(\lambda)^{2} \frac{\partial}{\partial \theta_{j}}\left\{\tilde{f}_{M, \theta_{M}}(\lambda)\right\}^{-1} \frac{\partial}{\partial \theta_{l}}\left\{\tilde{f}_{M, \theta_{M}}(\lambda)\right\}^{-1}\right\}_{\theta=\underline{\theta}_{M}} d \lambda\right. \\
\left.+2 \pi \iint_{\lambda_{0}-\frac{\pi}{2 M}}^{\lambda_{0}+\frac{\pi}{2 M}}\left\{\frac{\partial}{\partial \theta_{j}}\left\{\tilde{f}_{M, \theta_{M}}\left(\lambda_{1}\right)\right\}^{-1} \frac{\partial}{\partial \theta_{l}}\left\{\tilde{f}_{M, \theta_{M}}\left(\lambda_{2}\right)\right\}^{-1}\right\}_{\theta=\underline{\theta}_{M}} \widetilde{Q}^{Y}\left(-\lambda_{1}, \lambda_{2},-\lambda_{2}\right) d \lambda_{1} d \lambda_{2}\right] .
\end{gathered}
$$

To get a consistent estimator of $\tilde{f}^{*}\left(\lambda_{0}\right)$, we have to assume that $M \rightarrow \infty$ but $M=o(n)$. Then we obtain,

\section{Theorem 2.}

Suppose that $M=M(n) \rightarrow \infty$ and $\lim _{n \rightarrow \infty} \frac{M}{\sqrt{n}}=0$ as $n \rightarrow \infty$. Then, the following statement holds true.

$$
\sqrt{L}\left(\hat{\tilde{f}}_{M, \theta_{M}}^{*}\left(\lambda_{0}\right)-\tilde{f}^{*}\left(\lambda_{0}\right)\right) \stackrel{d}{\rightarrow} N\left(0, W_{\lambda_{0}}\right)
$$

where $W_{\lambda_{0}}=\frac{\sum_{i=-\infty}^{\infty} \sum_{j=-\infty}^{\infty} \varphi\left(\lambda_{0}+2 \pi i\right) \varphi\left(\lambda_{0}+2 \pi j\right)}{\left[\sum_{j=-\infty}^{\infty} \varphi\left(\lambda_{0}+2 \pi j\right)^{2}\right]^{2}} \times \tilde{f}\left(\lambda_{0}\right)^{2}$.

Theorem 1 implies that for sufficiently large fixed M, the local Whittle estimator $\hat{\boldsymbol{\theta}}_{M}$ has the asymptotic normality and $\sqrt{n}$-consistency and the asymp- 
totics depend on non-Gaussian quantities $\tilde{Q}^{Y}(\cdot, \cdot, \cdot)$. However, in Theorem 2, if we let $M \rightarrow \infty$ with $M=o(\sqrt{n})$, then the asymptotics of $\hat{\tilde{f}}_{M, \theta_{M}}^{*}\left(\lambda_{0}\right)$ are described by $\varphi(\cdot)$ and $\tilde{f}(\cdot)$, and are independent of non-Gaussianity(i.e., nonGaussian robust).

So far we have assumed that $\varphi(\lambda)$ is known. But, this assumption is not natural, hence, we suppose that $\varphi(\lambda)=\varphi_{\gamma}(\lambda), \gamma \in \mathcal{R}^{q}$, where $\gamma$ is unknown parameter, i.e., $\varphi_{\gamma}(\cdot)$ is a parametric model. From (2.7) we have the relation:

$$
\varphi_{\gamma}(\lambda)=\exp \{-i \lambda\} \cdot E\left\{e^{i\left(\Delta_{1} Z_{t}\right) \lambda}\right\}
$$

Suppose that an observed stretch $Z_{1}, Z_{2}, \cdots, Z_{n+1}$ is available, and let $W_{j} \equiv$ $\Delta_{1} Z_{j}, j=1, \cdots, n$. As in Knight and $\mathrm{Yu}(2002)$, we can introduce the following empirical characteristic function

$$
\hat{\varphi}_{n}(\lambda) \equiv \exp \{-i \lambda\} \frac{1}{n} \sum_{j=1}^{n} \exp \left\{i W_{j} \lambda\right\}
$$

for (3.16). Knight and Yu (2002) introduced an estimator of $\gamma$ by

$$
\hat{\gamma} \equiv \arg \min _{\gamma} \int_{-\pi}^{\pi}\left|\hat{\varphi}_{n}(\lambda)-\varphi_{\gamma}(\lambda)\right|^{2} d G(\lambda),
$$

where $G(\lambda)$ is an appropriate weight function, and showed the consistency and asymptotic normality of $\hat{\gamma}$ under natural regularity conditions. Therefore, in the case when $\varphi(\lambda)$ is unknown, we may use $\varphi_{\hat{\gamma}}(\lambda)$ in place of $\varphi(\lambda)$.

\section{Numerical Studies}

In this section we compare $\tilde{f}(\lambda)$ with $\hat{\tilde{f}}_{M, \theta_{M}}(\lambda)$ by simulation. We suppose that $\left\{Y_{t}\right\}$ is generated by the $\operatorname{AR}(1)$ model

$$
Y_{t}=\alpha Y_{t-1}+\epsilon_{t}
$$

where $\epsilon_{t} \sim$ i.i.d.N $(0,1)$ and the directing process $\left\{\Delta_{h} Z_{t}\right\} \sim$ i.i.d.N $(h, 1)$. For simplicity, in what follows we write $\lambda=\lambda_{0}$. Then, the spectral density of this model is

$$
\tilde{f}(\lambda)=\frac{1}{\left|1-\alpha e^{i \lambda}\right|^{2}} \times \frac{1}{2 \pi} .
$$

We set the length of observed stretch $Y_{t}$ is $n=100$ and $M=n^{\frac{1}{3}}$. Figures 1 and 2 plot the estimator $\hat{\tilde{f}}_{M, \theta_{M}}(\lambda)$ and the true spectral density for $\alpha=0.55,0.75$ respectively, where $\lambda=\frac{j-1}{50}, j=1, \cdots, 51$. Table 1 shows the MSE by 5,000 times simulation of $\hat{\tilde{f}}_{M, \theta_{M}}(\lambda)$ for $\alpha=0.55,0.75$ and $\lambda=\frac{\pi}{5}, \frac{2 \pi}{5}, \frac{3 \pi}{5}, \frac{4 \pi}{5}$. The comparison shows when the frequency $\lambda$ is low the estimator $\hat{\tilde{f}}_{M, \theta_{M}}(\lambda)$ is not so good. But as the frequency $\lambda$ becomes higher, the spectral estimator $\hat{\tilde{f}}_{M, \theta_{M}}(\lambda)$ becomes a good one. From the figures and table it seems interesting that goodness of the estimator $\hat{\tilde{f}}_{M, \theta_{M}}(\lambda)$ is frequency-dependent i.e., the estimator becomes a good one for high-frequencies. 


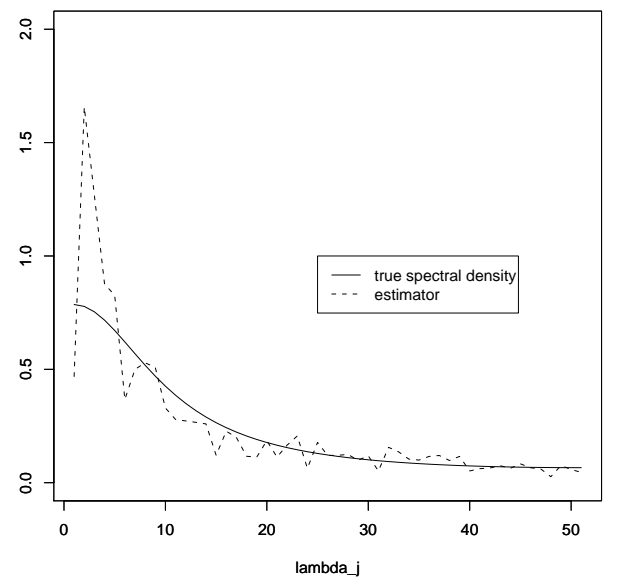

Figure 1: The spectral estimator $\hat{\tilde{f}}_{M, \theta_{M}}(\lambda)$ and the true spectral density $\tilde{f}(\lambda)$ of $\left\{Y_{t}\right\}(a=0.55)$.

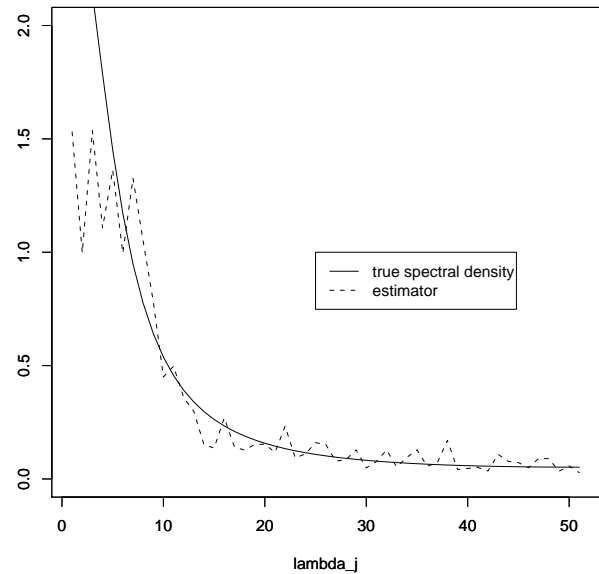

Figure 2: The spectral estimator $\hat{\tilde{f}}_{M, \theta_{M}}(\lambda)$ and the true spectral density $\tilde{f}(\lambda)$ of $\left\{Y_{t}\right\}(a=0.75)$. 


\begin{tabular}{|l||l|l|l|l|}
\hline$\lambda$ & $\frac{1}{5} \pi$ & $\frac{2}{5} \pi$ & $\frac{3}{5} \pi$ & $\frac{4}{5} \pi$ \\
\hline \hline$\alpha=0.55$ & 0.01399827 & 0.002447916 & 0.001011906 & 0.00050327 \\
\hline$\alpha=0.75$ & 0.04739601 & 0.002263211 & 0.000690388 & 0.000306882 \\
\hline
\end{tabular}

Table 1: MSE of the spectral estimator $\hat{\tilde{f}}_{M, \theta_{M}}(\lambda) \quad\left(\lambda=\frac{1}{5} \pi, \frac{2}{5} \pi, \frac{3}{5} \pi, \frac{4}{5} \pi\right)$ $(\alpha=0.55,0.75)$.

\section{Acknowledgements}

The authors thank Professor Hira Koul, an associate editor and a referee for their comments and kindness.

\section{References}

Anderson, T.W., 1971. The Statistical Analysis of Time Series. Springer, New York.

Brillinger, D.R., 1981. Time Series: Data Analysis and Theory. Holden Day, San Francisco.

Brockwell, P.J., Davis, R.A., 1991. Time Series: Theory and Methods. Springer, New York.

Clark, P.K., 1973. A Subordinated Stochastic Process Model with Finite Variance for Speculative Prices. Econometrica. 41, 135-156.

Ghysels, E., Gouriéroux, C., Jasiak, J., 1998. Kernel Autocorrelogram for Time Deformed Processes. J. Statist plan. \& Inf. 68, 167-191.

Hannan, E.J., 1970. Multiple Time Series. Wiley, New York.

Hosoya, Y., Taniguchi, M., 1982. A Central Limit Theorem for Stationary Processes and The Parameter Estimation of Linear Processes. Ann. Statist. 10, 132-153.

Knight, J.L., Yu, J., 2002. Empirical Characteristic Function in Time Series Estimation. Econometric Theory. 18, 691-721.

Mandelbrot, B., Taylor, H., 1967. On the distribution of stock prices differences. Oper. Res. 15, 1057-1062.

Stock, J., 1988. Estimating continuous time processes subject to time deformation. J. Amer. Statist. Assoc. 83(401) 77-84.

Taniguchi, M., Kakizawa, Y., 2000. Asymptotic Theory of Statistical Inference for Time Series. Springer, New York. 